\title{
Direct modulation of electroluminescence from silicon nanocrystals beyond radiative recombination rates
}

\author{
Josep Carreras, ${ }^{1, a)}$ J. Arbiol, ${ }^{1}$ B. Garrido, ${ }^{1}$ C. Bonafos, ${ }^{2}$ and J. Montserrat ${ }^{3}$ \\ ${ }^{1}$ EME-IN ${ }^{2}$ UB, Universitat de Barcelona, Martí i Franquès 1, 08028 Barcelona, Spain \\ ${ }^{2}$ nMat Group, CEMES-CNRS, 29 rue J. Marvig 31055, Toulouse Cedex 04, France \\ ${ }^{3}$ IMB-CNM, CSIC, 08193 Bellaterra, Spain
}

(Received 3 January 2008; accepted 6 February 2008; published online 3 March 2008)

\begin{abstract}
We propose a light emitting transistor based on silicon nanocrystals provided with $200 \mathrm{Mbits} / \mathrm{s}$ built-in modulation. Suppression of electroluminescence from silicon nanocrystals embedded into the gate oxide of a field effect transistor is achieved by fast Auger quenching. In this process, a modulating drain signal causes heating of carriers in the channel and facilitates the charge injection into the nanocrystals. This excess of charge enables fast nonradiative processes that are used to obtain 100\% modulation depths at modulating voltages of $\sim 1$ V. (C) 2008 American Institute of Physics. [DOI: 10.1063/1.2889499]
\end{abstract}

Silicon-based light emitting diodes ${ }^{1-4}$ (LEDs) and electro-optical modulators ${ }^{5-7}$ have recently shown potential for a monolithic integration of photonics and mainstream electronics. The majority of current silicon electro-optical modulators are based on plasma dispersion effects. ${ }^{7}$ Some reports conclude that they suffer from high power consumption (theoretical lower limit of current density of $\left.10^{4} \mathrm{~A} / \mathrm{cm}^{2}\right)^{6}$ and thus poor scalability. A metal-oxidesemiconductor field effect transistor (MOSFET)-based electroluminescent device would operate at lower power, merging the functionalities of an all-silicon LED and a high-speed modulator in a single transistor which can be readily integrated into silicon technology.

The devices studied in this work are MOSFETs with 100-nm-thick optically transparent polycrystalline silicon (polysilicon) gate electrodes and embedded silicon nanocrystals $(\mathrm{Si}-\mathrm{nc})$ in the gate oxide. The nanocrystals were obtained after $6.5 \mathrm{keV} \mathrm{Si}{ }^{+}$ion implantation into thermally grown $\mathrm{SiO}_{2}$ 15 -nm-thick layers and resulted in atomic Si peak excess of $20 \%$ at a projected range of $\sim 12 \mathrm{~nm}$, as simulated by SRIM (Ref. 8) code. The samples subsequently underwent an annealing in $\mathrm{N}_{2}$ at $1100{ }^{\circ} \mathrm{C}$ for $4 \mathrm{~h}$. A phosphorous implantation was used to define source and drain zones. A polysilicon layer was deposited by low-pressure chemical vapor deposition and degenerately doped with $\mathrm{POCl}_{3}$ to form a semitransparent gate electrode. Standard microelectronic processes of photolithography and etching were used to form the transistor structure.

The atomic silicon peak excess inside the matrix was confirmed to be $20 \%$ by x-ray photoelectron spectroscopy. The presence of Si-nc in the oxide was further verified by energy filtered transmission electron microscopy (EFTEM) at a silicon plasmon energy of $\sim 17 \mathrm{eV}$. Silicon nanocrystals were imaged at an average distance of $10.5 \mathrm{~nm}$ from the gate. The diameter of the observed nanoparticles was 4-5 nm.

Time-resolved electroluminescence (EL) was obtained with a detector (H7422P) and a photon counter (SR430). Electroluminescence spectra were obtained via a cryogenically cooled PI Spec-10-100B/LN charge-coupled device and

\footnotetext{
${ }^{a)}$ Electronic mail: carreras@el.ub.es.
}

an Acton 2300i grating spectrometer. Data were corrected with the overall optical transfer function.

The transistor structure resembles that of a Si-nc memory, ${ }^{9}$ having a floating gate embedded in the oxide made up of Si-nc. As schematically shown in Fig. 1(a), when a positive voltage is applied to the gate (i.e., $V_{G}$ $=5 \mathrm{~V})$, electrons from the inversion layer tunnel to the gate through Fowler-Nordheim (FN) injection, exciting Si-nc by impact. $^{2,10,11}$

The inset of Fig. 2(b) shows the EL spectrum obtained by this direct current (dc) excitation. The emission is centered at $\sim 725 \mathrm{~nm}$ and has a full width at half maximum of $\sim 220 \mathrm{~nm}$. Compared with bulk silicon, this represents an $\sim 0.6 \mathrm{eV}$ of gap expansion as a consequence of quantum

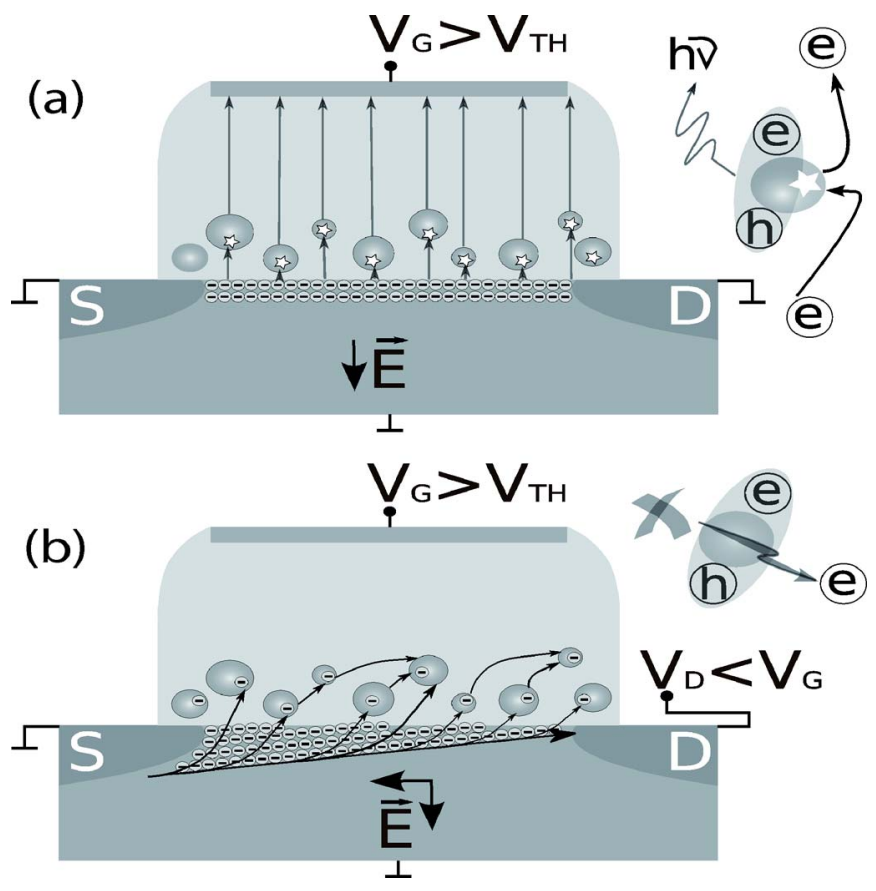

FIG. 1. (Color online) Injection processes schematics. (a) Si-nc exciton generation by impact excitation of electrons from the inversion layer through a direct-current gate-to-substrate voltage. (b) Schematic representation of the channel hot electron mechanism and subsequent electroluminescence quenching. 


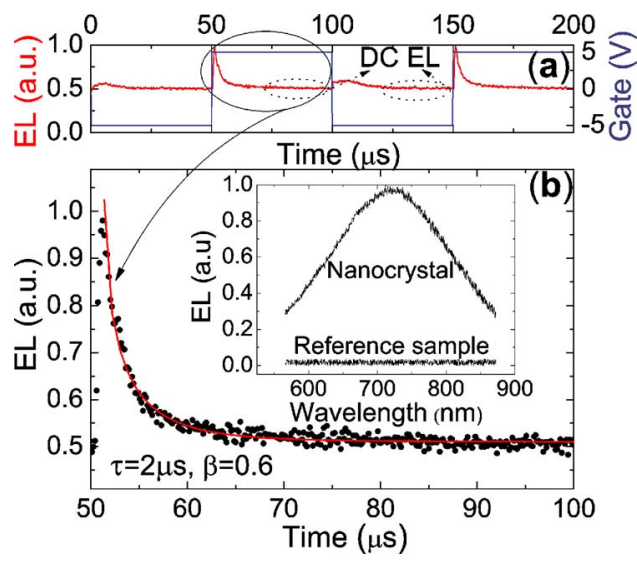

FIG. 2. (Color online) (a) Time-resolved electroluminescence through a $(5 \mathrm{~V}, 100 \mu \mathrm{s})$ square wave gate excitation. (b) Electroluminescence decay (dotted) and fitted data (solid). The inset shows the emission spectrum under $5 \mathrm{~V}_{\mathrm{DC}}$.

confinement, which corresponds ${ }^{12}$ to a population of excited Si-nc with an average size of $4-5 \mathrm{~nm}$, in accordance with EFTEM. Other studies ${ }^{3}$ attribute a $0.6 \mathrm{eV}$ gap expansion to smaller Si-nc $(\sim 2 \mathrm{~nm})$. Since EFTEM relies on changes within the targeted plasmon energy window and not on crystalline/amorphous contrast, the identification of a clear $\mathrm{Si} / \mathrm{SiO}_{2}$ edge is difficult, and nanocrystal sizes are usually overestimated. In addition, the measured transmittance pattern of the 100-nm-thick polysilicon contact peaks at $\sim 700 \mathrm{~nm}$, favoring a noticeable blueshift. This emission along with the microsecond-range radiative recombination times measured is a characteristic of exciton recombination in Si-nc due to quantum confinement effects. No emission was detected from control transistors without Si-nc.

Time-resolved EL decay measured in response to a $100 \mu$ s square wave applied to the gate is shown in Fig. 2(a). The EL peaks observed are attributed to a combination of (i) sequential exciton formation by injection of electrons (holes) from the inversion (accumulation) layer ${ }^{13-15}$ and (ii) impact excitation of carriers of different type. ${ }^{2,10}$ The observed decay [Fig. 2(b)] is well fitted by a stretched exponential function ${ }^{16}$ with $\tau=2 \mu \mathrm{s}(\beta=0.6)$. After $\sim 20 \mu \mathrm{s}$ of transient, the signal reaches its steady state; the device works through dc and shows a continuous EL (reduced by $\sim 50 \%$ from its initial peak value).

Electroluminescence modulation from an alternating current (ac) gate voltage beyond a few kilohertz has not yet been reported because it is inherently limited by three known mechanisms: ${ }^{2,14}$ tunneling times, Si-nc radiative lifetimes, and gate capacitance time constant. Under ac gate excitation, the maximum frequency for which EL modulation has been observed is about $50 \mathrm{kHz}$. This fundamental limitation imposed by the intrinsic nature of indirect-gap nanocrystals has relegated Si-nc to low-frequency applications. ${ }^{2,14}$

To overcome this limitation, our approach makes use of a MOSFET that combines dc gate excitation with an ac source-to-drain injection to obtain the EL quenching. This procedure enables the possibility to modulate light from Si-nc much faster than its physical limitation not by switching the excitation on/off but by activating/deactivating a nonradiative channel.

Channel-hot-electron (CHE) injection is widely used to program commercial NOR-type flash memories, and it is known to allow a fast injection and induce higher threshold Downloaded 24 Mar 2010 to 161.116.95.217. Redistribution subject
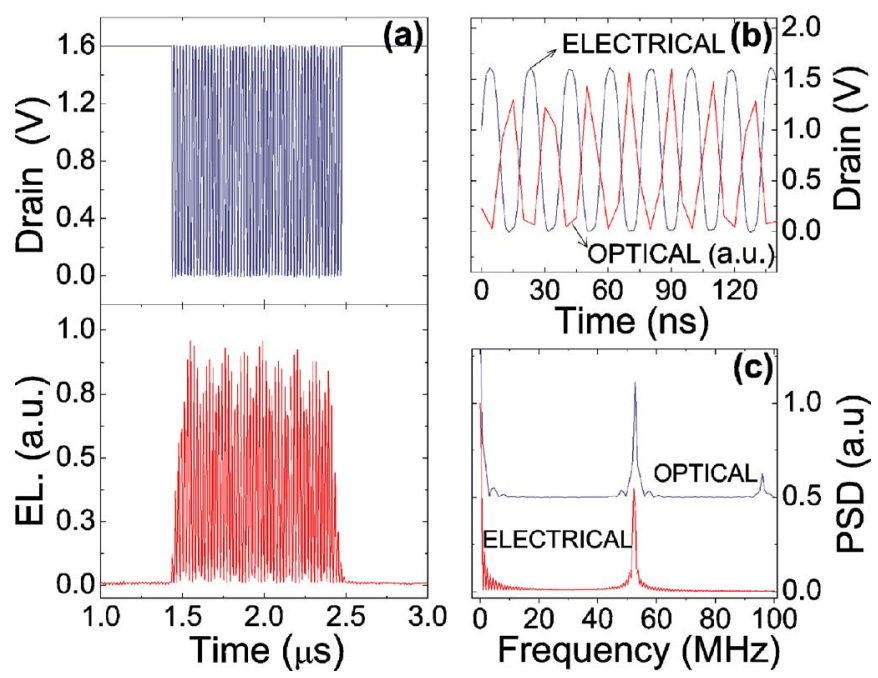

FIG. 3. (Color online) (a) Drain-induced electroluminescence modulation through mechanisms depicted in Fig. 1(b). (b) Close up of (a). (c) Power spectral density of the modulated (optical) and modulating (electrical) signals.

voltage shifts than FN programming. ${ }^{17}$ A modified CHE mechanism is schematically shown in Fig. 1(b). A constant gate voltage $\left(V_{G}>V_{\mathrm{TH}}\right.$, where $V_{\mathrm{TH}}$ is the threshold voltage $)$ causes light emission from nanocrystal excitons. A positive voltage is then applied to the drain $\left(V_{D}<V_{G}\right)$, which accelerates electrons from source to drain. This situation is well described by a heated non-Maxwellian energy distribution ${ }^{18}$ with a pronounced energy tail close to the drain. Provided with enough momentum direction toward the gate and a greater average energy, electrons are efficiently injected into the Si-nc layer.

An excess of charge in the Si-nc proximities drastically increases the Auger coefficient of the system, which, in turn, results in EL suppression. The nanocrystal regains its ability to emit a photon upon recovery of charge neutrality. The Auger mechanism has a subnanosecond decay time constant, ${ }^{19}$ being thus several orders of magnitude more effective than radiative recombination. The process is enhanced in nanocrystals compared to bulk because quantum confinement reinforces the Coulomb interaction between the charge carriers and relaxes the translational momentum conservation. $^{20}$

Figure 3(a) shows the observed modulation through drain cycling while the gate is fixed at $5 \mathrm{~V}$. Effective EL suppression is observed when the nanocrystal layer is flooded with electrons through CHE. Thus, we get rid of the most limiting factors of gate modulation. First, since the modulating signal is applied along a resistive path (MOSFET channel in inversion), capacitive delays are negligible. Second, modulation of Si-nc emission is governed by Auger times, so it is achieved at speeds several orders of magnitude faster than the Si-nc population radiative decay rates. Leaving aside Auger recombination, the performance of a device working through this principle would be ultimately limited by both the source-to-drain transistor bandwidth and the ability to inject/remove charge to/from the electroluminescent centers.

Figure 3(b) shows a detail of the modulated signal. The full width at half maximum of drain pulses is $10 \mathrm{~ns}$, corresponding to $100 \mathrm{MHz}$. A fast Fourier transform of data extracted from Fig. 3(a) is shown in Fig. 3(c), demonstrating
to AlP license or copyright; see http://apl.aip.org/apl/copyright.jsp 


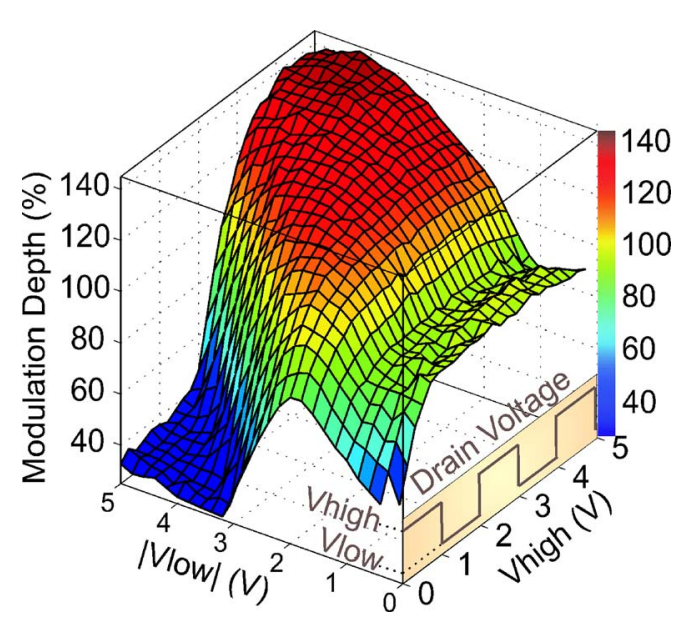

FIG. 4. (Color online) Calculated modulation depth over 625 drain $100 \mathrm{MHz}$ square waves versus the maximum and minimum value of the drain signal (see inset). The shape observed is coherently explained through injection mechanisms depicted in Figs. 1(a) and 1(b).

that information is not degraded by the modulation process. The time resolution of our photon-counting system is $5 \mathrm{~ns}$, and higher frequencies could not be tested in this work because of the equipment limitation.

Finally, we study the influence of a $100 \mathrm{MHz}$ modulation signal on the output emission. A set of 625 square waves differing in their positive/negative values $\left(V_{\text {high }}\right.$ and $V_{\text {low }}$, as depicted in the inset of Fig. 4) are sequentially applied to the drain, while the gate is biased at $5 \mathrm{~V}$. Time-resolved EL is measured on the same device for each wave form maintaining the same integration time and detector sensitivity. For each $\left(V_{\text {high }}, V_{\text {low }}\right)$ pair, the modulation depth (MD, defined as the ratio between the amplitude of the modulated signal and the constant optical signal level before modulation) is plotted in Fig. 4. A MD of about $95 \%$ is obtained for $\left(V_{\text {high }}, V_{\text {low }}\right)$ $=(1,0) \mathrm{V}$, indicating that drain voltages well below the gate bias are required to efficiently inject electrons through the thin tunnel oxide. For $V_{\text {low }}=0 \mathrm{~V}$ and $V_{\text {high }}>1 \mathrm{~V}$, pinch-off of the channel occurs and drain current no longer depends on drain voltage, which is manifested as a saturation of the MD. Although source and drain in a MOSFET are symmetrical terminals, $V_{\text {high }}$ and $V_{\text {low }}$ are not completely interchangeable as a high (negative) $V_{\text {low }}$ significantly increases the electric field in the oxide and strongly contributes to impact excitation from the drain region. When $V_{\text {high }}=0 \mathrm{~V}$ and $V_{\text {low }}>$ $-2 \mathrm{~V}, \mathrm{MD}$ is still acceptable $(\sim 80 \%)$ but rapidly decrease for $V_{\text {low }}<-2 \mathrm{~V}$, as a pinched-off channel cannot compensate the strong voltage dependence of a FN drain injection. It should be noted that MDs higher than $100 \%$ are measured for $\left(V_{\text {high }},\left|V_{\text {low }}\right|\right)>(2,2) \mathrm{V}$, again attributed to a suppression of signal while $V_{\text {high }}$ is applied, and an impact excitation enhancement or amplification taking place at $V_{\text {low }}$ as a consequence of an increased effective oxide field.

In conclusion, in this letter, we propose high-speed direct modulation of silicon EL in a device that deliberately exploits Auger relaxation to obtain modulation rates at least three orders of magnitude faster than $\mathrm{Si}$-nc radiative recombination rates. The modulation is achieved by combining $\mathrm{FN}$ impact (continous gate excitation) and $\mathrm{CHE}$ injection (source-to-drain charge injection), attributed to EL quenching through an Auger nonradiative channel. A reliable Si-nc light emitting transistor working at $100 \mathrm{MHz}$ (or $200 \mathrm{Mbits} / \mathrm{s}$ ) rate is demonstrated with $100 \%$ of modulation depths at modulating voltages as low as $\sim 1 \mathrm{~V}$. This work is a demonstration of principle, but the final speed attainable is only limited by the channel bandwidth and tunnel transparency of the thin tunnel oxide. In addition, since it is based on a MOSFET, high compatibility and scalability is guaranteed.

One of the authors (Josep Carreras) acknowledges support for sample fabrication in the Integrated Nano and Microelectronics Clean Room at IMB-CNM through the ICTS access program from the Spanish Ministry of Science and Education.

${ }^{1}$ A. Irrera, F. Iacona, I. Crupi, C. D. Presti, G. Franzó, C. Bongiorno, D. Sanfilippo, G. Di Stefano, A. Piana, P. G. Fallica, A. Canino, and F. Priolo, Nanotechnology 17, 1428 (2006).

${ }^{2}$ W. Skorupa, L. Rebohle, and T. Gebel, Appl. Phys. A: Mater. Sci. Process. 76, 1049 (2003).

${ }^{3}$ O. Jambois, H. Rinnert, X. Devaux, and M. Vergnat, J. Appl. Phys. 98, 046105 (2005).

${ }^{4}$ O. Jambois, J. Carreras, A. Pérez-Rodríguez, B. Garrido, C. Bonafos, S. Schamm, and G. Ben Assayag, Appl. Phys. Lett. 91, 211105 (2007).

${ }^{5}$ A. Liu, R. Jones, L. Liao, D. Samara-Rubio, D. Rubin, O. Cohen, R. Nicolaescu, and M. Paniccia, Nature (London) 427, 615 (2004).

${ }^{6}$ L. Gu, W. Jiang, X. Chen, L. Wang, and R. T. Chen, Appl. Phys. Lett. 90, 071105 (2007).

${ }^{7}$ V. R. Almeida, Q. Xu, and M. Lipson, Opt. Lett. 30, 2403 (2005).

${ }^{8}$ J. F. Ziegler, J. P. Biersack, and U. Littmark, The Stopping and Range of Ions in Solids (Pergamon, New York, 1985).

${ }^{9}$ H. Hanafi, S. Tiwari, and I. Khan, IEEE Trans. Electron Devices 43, 1553 (1996).

${ }^{10}$ A. Irrera, D. Pacifici, M. Miritello, G. Franzò, F. Priolo, F. Iacona, D. Sanfilippo, G. Di Stefano, and P. G. Fallica, Appl. Phys. Lett. 81, 1866 (2002).

${ }^{11}$ O. Jambois, B. Garrido, P. Pellegrino, J. Carreras, A. Pérez-Rodríguez, J. Montserrat, C. Bonafos, G. Ben Assayag, and S. Schamm, Appl. Phys. Lett. 89, 253124 (2006).

${ }^{12}$ L. Ding, T. P. Chen, Y. Liu, M. Yang, J. I. Wong, Y. C. Liu, A. D. Trigg, F. R. Zhu, M. C. Tan, and S. Fung, J. Appl. Phys. 101, 103525 (2007).

${ }^{13}$ R. J. Walters, G. I. Bourianoff, and H. A. Atwater, Nat. Mater. 4, 143 (2005).

${ }^{14}$ R. J. Walters, J. Carreras, T. Feng, L. D. Bell, and H. A. Atwater, IEEE J. Sel. Top. Quantum Electron. 12, 1647 (2006).

${ }^{15}$ M. Perálvarez, C. García, M. López, B. Garrido, J. Barreto, C. Domínguez, and J. A. Rodríguez, Appl. Phys. Lett. 89, 051112 (2006).

${ }^{16}$ J. Linnros, N. Lalic, A. Galeckas, and V. Grivickas, J. Appl. Phys. 86, 6128 (1999).

${ }^{17}$ G. Ammendola, V. Ancarani, V. Triolo, M. Bileci, D. Corso, I. Crupi, L. Perniola, C. Gerardi, S. Lombardo, and B. DeSalvo, Solid-State Electron. 48, 1483 (2004).

${ }^{18}$ A. Gehring, T. Grasser, H. Kosina, and S. Selberherr, J. Appl. Phys. 92, 6019 (2002).

${ }^{19}$ R. M'ghaïeth, H. Maâref, I. Mihalcescu, and J. C. Vial, Phys. Rev. B 60, 4450 (1991).

${ }^{20}$ C. Delerue, G. Allan, and M. Lannoo, Light Emission in Silicon: From Physics to Devices, Semiconductors and Semimetals Vol. 49 (Academic, New York, 1998), p. 253. 